\title{
Detection of mycobacteria in bone marrow biopsy specimens taken to investigate pyrexia of unknown origin
}

\author{
U B G Riley, S Crawford, S P Barrett, S H Abdalla
}

\begin{abstract}
Aims-To investigate the value of bone marrow biopsy in the diagnosis of mycobacterial infection.

Methods-The culture results of 433 bone marrow samples taken between 1983 and 1992 were reviewed. The histopathology reports on bone marrow trephine specimens of culture positive samples and all those on HIV positive patients sent in 1992 were also reviewed.
\end{abstract}

Results-Fifty one specimens yielded Mycobacterium spp, 47 were obtained from HIV positive patients. Of the isolates, 42 were Mycobacterium avium-intracellulare (MAI), five were $M$ tuberculosis (MTB), and the remaining four comprised a variety of atypical mycobacteria. All MAI positive samples were obtained from HIV positive patients, with the bone marrow being the only culture positive specimen in one third. Bone marrow yielded MTB only in patients from whom it was also isolated in other specimens. Eleven of 47 trephine specimens from positive bone marrow showed granulomata and nine showed acid-fast bacilli. No acid-fast bacilli were seen in the absence of granulomata.

Conclusion-Bone marrow biopsy for mycobacterial culture should be reserved for severely immunosuppressed patients and should not be advocated for immunocompetent patients with suspected tuberculosis. Bone marrow biopsy still has a role in the investigation of pyrexia of unknown origin in HIV positive patients, despite the advent of mycobacterial blood culture techniques, particularly if these can be processed safely in automated systems. (f Clin Pathol 1995;48:706-709)

Keywords: Mycobacteria, HIV, pyrexia of unknown origin.

Bone marrow biopsy has been widely used for the investigation of pyrexia of unknown origin (PUO) in both the immunocompetent and immunocompromised patient. ${ }^{12}$ Mycobacteria have been considered an important cause of
PUO, particularly Mycobacterium avium-intracellulare (MAI) in patients infected with $\mathrm{HIV}^{3}$ and they can disseminate to the bone marrow in patients with AIDS. The value of bone marrow biopsy has been questioned in the investigation of PUO. ${ }^{5}$ For investigation of mycobacterial infection in HIV positive patients, methods other than bone marrow biopsy-for example, blood cultures and liver biopsy, have been used for their ease and rapid results. ${ }^{4-6}$ We have analysed retrospectively the results of 433 bone marrow samples sent for mycobacterial culture between 1983 and 1992 at the St Mary's group of hospitals in London. We have looked at the change in isolation rates of mycobacteria during this period and have attempted to evaluate the importance of bone marrow compared with other specimen types in the investigation of mycobacterial infection in PUO.

\section{Methods}

Bone marrow specimens $(n=433)$ were cultured over a 10 year period, of which 234 were from HIV positive patients. All bone marrow samples were collected by a clinical haematologist. Bone marrow biopsy in the HIV positive patients was usually prompted by an unexplained fever of at least one week's duration, as illustrated by a review of indications for bone marrow biopsies in 1992 (table 1). HIV positive patients were at various stages of HIV infection, ranging from patients with uncomplicated HIV seropositivity to frank AIDS. Culture for mycobacteria was the main microbiological reason for requesting bone marrow examination, but occasionally samples were also sent for examination for fungi and Leishmania spp. Patients who were not HIV positive had mycobacterial bone marrow examination when disseminated $M$ tuberculosis (MTB) was strongly suspected as the cause of PUO, or when a covert mycobacterial infection was thought to be associated with a haematological abnormality or suspected lymphoma.

STAINING

Bone marrow smears were made on glass slides by the haematologist at the bedside and were 
Table 1 Indications for bone marrow biopsy in $102 \mathrm{HIV}$ positive patients during 1992

\begin{tabular}{lcc}
\hline & No. of patients & Per cent \\
\hline PUO & 70 & $68 \cdot 6$ \\
Suspected lymphoma/staging & 9 & $8 \cdot 8$ \\
Anaemia & 11 & $10 \cdot 8$ \\
Thrombocytopenia & 1 & 1 \\
Splenomegaly & 2 & 2 \\
Lymphadenopathy & 9 & $8 \cdot 8$ \\
\hline
\end{tabular}

Table 2 Isolation of mycobacteria from bone marrow biopsy specimens between 1983 and 1992

\begin{tabular}{lcll}
\hline & & \multicolumn{2}{l}{$\begin{array}{l}\text { No. of isolates with patient HIV } \\
\text { status (\%) }\end{array}$} \\
\cline { 3 - 4 } Year & No. of samples & Positive & Negative \\
\hline 1983 & 21 & $0 / 0(0)$ & $0 / 21(0)$ \\
1984 & 24 & $0 / 4(0)$ & $0 / 20(0)$ \\
1985 & 25 & $1 / 3(33 \cdot 3)$ & $0 / 22(0)$ \\
1986 & 19 & $0 / 7(0)$ & $0 / 12(0)$ \\
1987 & 29 & $2 / 12(16 \cdot 7)$ & $0 / 17(0)$ \\
1988 & 25 & $0 / 17(0)$ & $0 / 8(0)$ \\
1989 & 29 & $4 / 18(22 \cdot 2)$ & $0 / 11(0)$ \\
1990 & 49 & $6 / 33(18 \cdot 2)$ & $0 / 16(0)$ \\
1991 & 71 & $8 / 50(16)$ & $0 / 21(0)$ \\
1992 & 139 & $26 / 90(32)$ & $2 / 49(8 \cdot 2)$ \\
Total & 433 & $47 / 234(20 \cdot 1 \%)$ & $4 / 199(2 \%)$ \\
\hline
\end{tabular}

sent to the laboratory with culture specimens. They were then stained with auraminerhodamine. Bone marrow trephine specimens were processed in the histopathology department. They were fixed, decalcified, and slides were made and routinely stained with haemotoxylin and eosin and Wright-Giemsa stain. On request, or routinely in the case of HIV positive patients, Ziehl-Neelsen and periodic acid/Schiff (PAS) stains were used to detect mycobacteria and fungal bodies, respectively.

\section{CULTURE}

Before January 1991, bone marrow was directly inoculated onto Lowenstein-Jensen (LJ) slope medium by the haematologist and sent to the laboratory. Excess bone marrow was then inoculated into Kirchner's liquid medium. Because of leakage from $\mathrm{LJ}$ slope jars, from January 1991 bone marrow samples were inoculated directly into 12B BACTEC bottles and sent to the laboratory. All cultures were incubated for eight weeks at $37^{\circ} \mathrm{C}$; $\mathrm{LJ}$ and Kirchner's media were cultured for a further four weeks for HIV positive patients. All cultures were checked weekly for evidence of growth; 12B BACTEC bottles were run in the BACTEC 460-TB system (Becton Dickinson, Oxford, UK). The presence of mycobacteria was confirmed by auramine-rhodamine and Ziehl-Neelsen staining.

\section{IDENTIFICATION}

All positive cultures were subcultured on to $\mathrm{LJ}$ slopes and sent to the Mycobacterium Reference Unit, University Hospital of Wales, Cardiff, for identification and sensitivity testing.

OTHER SAMPLES

Most patients had a variety of additional specimens collected for the investigation of PUO, including others for mycobacterial culture. Most of these samples were collected within 21 days of the bone marrow sample. These ranged from easily obtainable samples, including sputum and early morning urine, to those requiring invasive procedures, such as bronchoalveolar lavage and liver biopsy.

From August 1986 to May 1992, 231 blood cultures were taken from a number of HIV positive patients as part of a prospective study. ${ }^{7}$ These were inoculated into Dupont paediatric blood culture tubes which contained saponin to lyse macrophages. These were inoculated, without centrifugation, into Kirchner's liquid medium containing amphotericin B and polymixin, and also into Middlebrook 7H9 broth base. They were cultured for eight weeks at $37^{\circ} \mathrm{C}$. Routine mycobacterial blood cultures were not performed during the period of the review.

\section{Results}

Between 1983 and 1992, 433 bone marrow samples were cultured for mycobacteria which were detected in $51(11.7 \%)$ of them. None of the culture positive samples had been positive on auramine-rhodamine staining. During the first six years, three $(2 \cdot 1 \%)$ of 144 samples were positive compared with $48(16.6 \%)$ of 289 samples in the following four years (table 2). The 51 isolates came from 45 patients (tables 2 and 3$)$. Of these, $41(91 \cdot 1 \%)$ were known to be HIV positive. Of the remaining patients, one child with undetermined immunodeficiency was known to be HIV negative; the other three were not formally tested, but were not thought to be at high risk of HIV infection.

\section{ISOLATES}

The results from 45 patients with positive bone marrow cultures are shown in table 3. MAI accounted for $42(82.3 \%)$ of the 51 isolates that were obtained from 36 patients, all of whom were HIV positive. In 12 (33.3\%) patients bone marrow was the only positive culture for MAI; three of these patients had no
Table 3 Mycobacterial species isolated from bone marrow biopsy specimens*

\begin{tabular}{lcclc}
\hline & $\begin{array}{l}\text { Total no. of } \\
\text { isolates }\end{array}$ & $\begin{array}{l}\text { No. from HIV } \\
\text { positive patients }\end{array}$ & $\begin{array}{l}\text { No. from HIV } \\
\text { negative patients }\end{array}$ & $\begin{array}{l}\text { No. of patients with bone } \\
\text { marrow isolates only }\end{array}$ \\
\hline MTB & 5 & 3 & 2 & 0 \\
MAI & 42 & 36 & 0 & 12 \\
M kansasii & 2 & 1 & 1 & 1 \\
M xenopi & 1 & 1 & 0 & 0 \\
M fortuitum & 1 & 0 & 1 & 0 \\
Total & 51 & 41 & 4 & 13 \\
\hline
\end{tabular}

* Some patients yielded more than one positive isolate.
Table 4 Patients with negative bone marrow cultures and mycobacteria isolated from other sites

\begin{tabular}{lcll}
\hline Isolate & No. of isolates & $\begin{array}{l}\text { No. from HIV } \\
\text { positive patients }\end{array}$ & $\begin{array}{l}\text { No. from HIV } \\
\text { negative patients }\end{array}$ \\
\hline MTB & 8 & 2 & 6 \\
MAI & 2 & 2 & 0 \\
Total & 10 & 4 & 6 \\
\hline
\end{tabular}


other specimens submitted for mycobacterial examination. Twenty four patients had other samples positive for MAI apart from bone marrow. In 10 of these patients the bone marrow was the first sample to provide a bacteriological indication of mycobacterial infection. There was a wide variety of other samples that were culture positive; liver biopsy (nine isolates), rectal biopsy (five isolates) and early morning urine (five isolates) being the most common.

MTB accounted for five $(9.8 \%)$ isolates; three were from HIV positive patients who had MTB isolated from pulmonary sites. In one patient MTB was also isolated from ascitic fluid and a lymph node biopsy. The two remaining patients had MTB isolated from ascitic fluid and pleural aspirate samples.

There were two isolates of $M$ kansasii, one from a HIV positive patient with MAI also isolated from other sites, the other from a patient with terminal hairy cell leukaemia. $M$ xenopi was isolated from bone marrow and bronchoalveolar lavage specimens of a HIV positive patient. $M$ fortuitum was isolated from the child with an unclassified immunodeficiency and was also recovered from swabs of a chest wall sinus.

Between 1983 and 1992, there were 10 patients with negative bone marrow cultures but who had mycobacteria isolated from other sites (table 4). Four of the patients were HIV positive; eight isolates were of MTB and two were of MAI.

\section{COMPARISON OF MYCOBACTERIAL BLOOD} CULTURES WITH BONE MARROW CULTURE

Two hundred and thirty one samples were received for mycobacterial blood culture from 143 patients, 142 of whom were HIV positive and one was the child with an undetermined immunodeficiency syndrome. Twenty two $(9.5 \%)$ cultures were positive and were obtained from 19 patients.

Thirty patients had both blood cultures and bone marrow cultures performed and comparative results are shown in table 5 .

Table 5 Results of mycobacterial isolation in patients by both blood and bone marrow cultures*

\begin{tabular}{lcccc}
\hline & & \multicolumn{3}{c}{ Mycobacteria from blood } \\
\cline { 2 - 4 } & & Yes & No & \\
\hline Mycobacteria from bone marrow & Yes & 10 & 3 & 13 \\
& No & 1 & 16 & 17 \\
& & 11 & 19 & 30
\end{tabular}

* All isolates were MAI except one isolate of $M$ fortuitum isolated from bone marrow and not from blood culture.

Table 6 Comparison of bone marrow culture and bone marrow trephine specimen examination

\begin{tabular}{lllll}
\hline Isolate & Total no. & $\begin{array}{l}\text { No. from HIV } \\
\text { positive patients }\end{array}$ & $\begin{array}{l}\text { Granuloma } \\
\text { present }\end{array}$ & $\begin{array}{l}\text { Acid-fast bacilli } \\
\text { present }\end{array}$ \\
\hline MAI & 41 & 41 & 10 & 8 \\
MTB & 4 & 3 & 0 & 0 \\
M xenopi & 1 & 1 & 0 & 0 \\
M kansasii & 1 & 0 & 1 & 1 \\
Total & 47 & 45 & 11 & 9 \\
\hline
\end{tabular}

MICROSCOPY OF BONE MARROW TREPHINE SPECIMENS

Of the 51 culture positive specimens collected in 10 years, bone marrow trephine results were available on 47,45 from HIV positive patients (table 6). Acid-fast bacilli were only seen in conjunction with granulomata. The trephine specimens from HIV positive patients in 1992 were reviewed separately. One hundred and three bone marrow biopsies were performed on HIV positive patients with 88 biopsy specimens sent for histopathological investigation; granulomata were reported in $11(12.5 \%)$ of these and in six of the patients with granulomata acid-fast bacilli were also reported. In one patient large numbers of granulomata and acidfast bacilli were seen in the trephine specimen but these were not grown from an aspirate sent in a BACTEC bottle. Acid-fast bacilli were also seen in a duodenal biopsy specimen from this patient examined histopathologically. Blood cultures performed as part of the above mentioned study were negative. Six stools collected from this patient were positive for acid-fast bacilli by auramine staining, but from only one was MAI isolated. One other patient had two bone marrow biopsy specimens taken which showed loose aggregates of histiocytes but no acid-fast bacilli; both isolates subsequently grew MAI.

Of the remaining five patients where granulomata but no acid-fast bacilli were reported, one subsequently grew MAI. One patient's trephine specimen revealed yeast bodies confirmed as Cryptococcus spp. Two patients had cytomegalovirus infection: one with retinitis, the other with hepatitis and colitis; neither had evidence of cytomegalovirus involvement of the bone marrow. The remaining patient had noncaseating granuloma in both bone marrow and liver biopsy specimens. No organism was isolated from these samples and the patient was diagnosed as having Pneumocystis carinii pneumonia when specimens were collected.

\section{Discussion}

In our experience, bone marrow biopsy has become increasingly popular in the investigation of PUO, particularly in HIV positive patients. Acid-fast bacilli were not seen in stained smears of bone marrow aspirates, even in those patients who had acid-fast bacilli on staining of the trephine specimen. This differs from other studies ${ }^{89}$ where a small, but measurable, percentage of mycobacteria were seen in the bone marrow aspirates. This could be because of two factors: concentration of the marrow by centrifugation in one study ${ }^{8}$ may have increased the sensitivity compared with direct smears, and the use of additional microscopic techniques in combination with direct smears in the other study ${ }^{9}$ may also have increased the rate of positivity.

The low percentage of granulomata in culture positive patients $(24.4 \%)$ in our study supports the findings of others ${ }^{10}$ and indicates that examination of trephine specimens may predict mycobacterial infection less reliably than liver biopsy. ${ }^{56}$ Acid-fast bacilli were seen only in the presence of granulomata, a finding re- 
flected in other studies, ${ }^{831112}$ and suggests that it may be unnecessary to use selective stains for mycobacteria if no granulomata are seen.

The finding that, in one year, $12.5 \%$ of trephine examinations in HIV positive patients had granulomata, contrasts with the higher value of $30 \%$ in the study by Nichols et $a l^{8}$ which we feel can be partly explained by retrospective re-examination of trephine slides and the classification of loose aggregates of histiocytes and other subtle findings as granulomata in that study. Our review of bone marrow biopsy specimens with granulomata in HIV positive patients from 1992 , showed that $72 \cdot 7 \%$ had microscopic or cultural evidence of bone marrow infection and that all patients with granulomata had evidence of opportunistic infection. This indicates that the presence of granulomata in the bone marrow of HIV positive patients is highly suggestive of infection. The finding of one patient with abundant granulomata and acid-fast bacilli on microscopy but who was culture negative was particularly interesting. It could be explained by the use of antimicrobials with some anti-mycobacterial action during the course of his PUO, or by infection with the recently described mycobacteria which has been termed Mycobacterium genavense- this bacterium does not grow on conventional solid media and grows poorly in liquid media. ${ }^{13}$ Although our review confirms that culture is more sensitive than microscopy for investigation of mycobacterial infection, examination of trephine specimens may provide indirect evidence of opportunistic infection if granulomata are present, and diagnosis of organisms which are difficult to culture.

In the immunocompetent patient with PUO, disseminated MTB is often suspected. Although our review confirmed that MTB can be recovered from the immunocompetent, it was isolated in only five cases in the 10 year period; in all five cases MTB was also isolated from other sites. Of the eight patients who had proven MTB infection but negative bone marrow cultures, six were HIV negative. This would suggest that in suspected disseminated MTB infection in HIV negative patients, involvement of bone marrow is uncommon and that bone marrow biopsy for disseminated MTB may not be worthwhile.

MAI was the predominant mycobacterial species isolated from bone marrow during the 10 year period of the review. Bone marrow is a useful method of confirming disseminated MAI disease, as mycobacteria in bone marrow indicate infection, in contrast to isolation from the gut-for example, which may indicate colonisation. The value of bone marrow culture in the diagnosis of MAI is demonstrated by MAI being isolated from the bone marrow alone in one quarter of our patients, whereas MAI was not isolated from bone marrow when cultured from other sites in only two patients. Direct comparison between blood cultures and bone marrow biopsy was possible in some cases which did not show substantial differences be- tween the two methods, and showed that neither method detected all cases of disseminated mycobacterial disease. This observation has been made in other studies. ${ }^{810}$ However, blood culture has the advantage that it is minimally invasive with little associated morbidity permitting repeated sampling.

In conclusion, our review of bone marrow culture and examination of trephine specimens leads us to believe that routine bone marrow biopsy for PUO should be reserved for those with severe immunosuppression. In subjects with normal immunity, MTB is the only mycobacterium likely to be recovered from bone marrow, and this may be more readily isolated from other sites if disseminated. The finding in some patients of bone marrow cultures positive for MAI despite all other samples, including blood cultures, being negative, and the potential for the diagnosis of other infectious and non-infectious causes of PUO, lead us to believe that bone marrow still has a valuable place in the investigation of PUO in HIV positive patients. We suggest that the correct approach is to take mycobacterial blood cultures when the patient presents with simple fever, and if these remain negative after two to three weeks, proceed to bone marrow biopsy.

We would like to thank Dr Ivor Brown, Histopathology Department, and Alistair Hill and Suzie Bailey, Department of Genito-Urinary Medicine, St Mary's Hospital, London, for their help in gathering the data for this paper.

1 Jacoby GA, Swark MN. Fever of undetermined origin. $N$ Engl ₹ Med 1973;289:1407-10.

2 Chaisson RE, Schecter FG, Theuer CP, Rutherford GW, Echenberg DF, Hopewell PC. Tuberculosis in patients with the acquired immunodeficiency syndrome. Am Rev Respir Dis 1987;136:570-4.

3 Karcher DS, Frost AR. The bone marrow in human immunodeficiency virus (HIV)-related disease: morphology and clinical correlation. Am f Clin Pathol 1991;95:63-71.

4 Macher AM, Kovacs JA, Gill V, Roberts GD, Ames J, Park $\mathrm{CH}$, et al. Bacteremia due to Mycobacterium aviumintracellulare in the acquired immunodeficiency synintracellulare in the ann Intern Med 1984;101:644-5.

5 Prego V, Glatt AE, Roy V, Thelmo W, Dincsoy H, Raufman J-P. Comparative yield of fungi and mycobacteria, liver biopsy, and bone marrow biopsy in Human Immunodeficiency Virus-infected patients. Arch Intern Med 1990; 50:333-6.

6 Cappell C, Schwartz MS, Biempica L. Clinical utility of liver biopsy in patients with serum antibodies to the Human Immunodeficiency Virus. Am f Med 1990;88: 123-30.

7 Hellyer TJ, Brown IN, Taylor MB, Allen BW, Easmon CSF. Gastrointestinal involvement in Mycobacterium aviumintracellula

8 Nichols L, Florentine B, Lewis W, Sattler F, Rarick MU, Byrnes RK. Bone marrow examination for the diagnosis of mycobacterial and fungal infections in the acquired immunodeficiency syndrome. Arch Pathol Lab Med 1991; 115:1125-32.

9 Uribe-Botero G, Prichard JG, Kaplowitz HJ. Bone marrow in HIV infection-A comparison of fluorescent staining and cultures in the detection of mycobacteria. $A m \mathcal{F} \mathrm{Clin}$ Pathol 1989;91:313-15.

10 Bishburg E, Eng RHK, Smith SM, Kapila R. Yield of bone marrow culture in the diagnosis of infectious diseases in patients with Acquired Immunodeficiency Syndrome. $\mathcal{F}$ Clin Microbiol 1986;24:312-14.

11 Castella A, Croxson TS, Mildvan D, Witt DH, Zalusky R. The bone marrow in AIDS. Am $\mathcal{F}$ Clin Pathol 1985;84 425-32.

12 Gluckman RJ, Rosner F, Guarneri JJ. The diagnostic utility of bone marrow aspiration and biopsy in patients with acquired immunodeficiency syndrome. $\mathcal{F}$ Natl Med Assoc 1989;81:119-25.

13 Bottger E, Teske A, Kirschner P, Bost S, Change HR, infection in patients with AIDS. Lancet 1992;340:76-80. 